\title{
Comparative performances of DNA barcoding across insect orders
}

\author{
Massimiliano Virgilio*1,2, Thierry Backeljau'2,3, Bruno Nevado² and Marc De Meyer ${ }^{1}$
}

\begin{abstract}
Background: Previous studies on insect DNA barcoding provide contradictory results and suggest not consistent performances across orders. This work aims at providing a general evaluation of insect DNA barcoding and "minibarcoding" by performing simulations on a large database of 15,948 DNA barcodes. We compared the proportions of correctly identified queries across a) six insect orders (Coleoptera, Diptera, Hemiptera, Hymenoptera, Lepidoptera and Orthoptera), b) four identification criteria (Best Match: BM; Best Close Match: BCM; All Species Barcodes: ASB; tree-based identification: NJT), and c) reference databases with different taxon coverage (100, 500, 1,000, 1,500 and 1,995 insect species).

Results: Analysis of variance revealed highly significant differences among ID criteria and insect orders. A posteriori comparisons of means showed that NJT had always a significantly lower identification success (NJT = 0.656, S.D. $=$ $0.118)$ compared to both $B M$ and $B C M(B M=0.948$, S.D. $=0.026 ; B C M=0.946, S . D .=0.031)$. NJT showed significant variations among orders, with the highest proportion of correctly identified queries in Hymenoptera and Orthoptera and the lowest in Diptera. Conversely, the proportions of correct matches of BM and BCM were consistent across orders but a progressive increase in false identification was observed when larger reference databases were used.

Conclusions: Regardless the relatively low proportion of Type I errors (misidentification of queries which are represented in the reference database) of BM and BCM, the lack of reference DNA barcodes for $98 \%$ of the known insect species implies that insect DNA barcoding is heavily biased by Type II errors (misidentification of queries without conspecifics in the database). The detrimental effects of Type II errors could be circumvented if insect DNA barcoding is used to verify the lack of correspondence between a query and a list of properly referenced target species (e.g. insect pests). This "negative identification" would only be subjected to Type I errors and could be profitably adopted in insect quarantine procedures.
\end{abstract}

\section{Background}

DNA barcoding aims at identifying organisms by assessing their degree of DNA sequence similarity to a set of reference taxa. The standard sequence used for this purpose is the mitochondrial COI gene fragment amplified by the "universal primers" of Folmer et al. [1]. Sequence similarities are then interpreted using numerical methods such as hierarchical clustering of genetic distances and statistical evaluations of genetic distance thresholds [2]. Recently, DNA barcoding has been explicitly defined as the molecular identification of a species based on the reference sequence with the lowest genetic distance [3]. Yet,

* Correspondence: massimilano.virgilio@africamuseum.be

1 Royal Museum for Central Africa, Leuvensesteenweg 13, 3080, Tervuren, Belgium

Full list of author information is available at the end of the article other numerical methods have been proposed to improve this approach [4-7].

DNA barcoding is generally considered as a reliable, cost-effective and easy molecular identification tool with a wide applicability across metazoan taxa [8-12]. As such it could be very useful to routinely identify difficult taxa of economic and medical importance. This particularly holds for many insect taxa that comprise large numbers of notorious pest species or disease vectors, whose identification often requires highly specialised taxonomic skills. In addition, DNA barcoding could be pivotal for the identification of certain life stages (e.g. eggs, larvae, nymphs or pupae), which are often impossible to identify otherwise. However, despite these highly positive claims, DNA barcoding also seems to suffer from a number of potential limitations when used for the identification of 
insects [4]. The recent speciation, the prevalence of paraphyly and the regular interspecific hybridisation in many insect taxa, as well as their often poorly-established taxonomy and their high degree of infection by endosymbiotic bacteria such as Wolbachia [11,13-15] may all negatively affect the performance of insect DNA barcoding. Even more importantly, the reliability of insect DNA barcoding may be questioned because insects include $>1,000,000$ described species and probably millions of still undescribed taxa [15]. This exuberant species richness may, indeed, severely constrain the ability of the DNA barcode reference databases to adequately represent the overwhelming insect taxonomic diversity.

Not surprisingly, insect DNA barcoding has hitherto produced contradictory results. Several studies showed that it is a reliable tool for the molecular identification of Lepidoptera [8,12,16], Hymenoptera [11,17], Coleoptera [18] and Diptera species $[19,20]$. Yet, other studies questioned the adequacy of DNA barcoding in Lepidoptera [21] and Orthoptera [22], while Meier et al. [4] reported a remarkably low identification success for Diptera $(<70 \%$ in simulations based on $>400$ taxa). The limited success of DNA barcoding evidenced by Meier et al. [4] was attributed to the use of GenBank sequences, which supposedly include a high proportion of misidentified sequences [23]. A closer look at the data of Meier et al. [4] shows that 322 out of 449 species in their dataset (corresponding to approximately $72 \%$ of taxa and $24 \%$ of sequences) are represented by single DNA barcodes. When these DNA barcodes are used as queries in simulations, they necessarily generate incorrect identifications, because there are no other conspecific reference sequences in the dataset with which they can match [24]. Meier et al. [4] included species represented by single barcodes in order to better reflect real-life situations, where it is not possible to know in advance if a query has a conspecific match in the database. However, this approach does not allow distinguishing the negative effects of poor taxon coverage from other potential constraints on insect DNA barcoding.

An additional problem with insect DNA barcoding is that the reference databases strongly rely on DNA sequencing of museum material, the success of which is often limited $[25,26]$. The DNA quality of museum specimens is generally low as it rapidly degrades over time. For example, less than $50 \%$ of moth and wasp museum specimens that were fixed for $<8$ years still yielded useful DNA barcodes [26]. The use of more efficient DNA extraction and repair protocols, and the amplification of multiple overlapping sections of the barcode region, generally improves the DNA barcoding success rates, though at a substantially higher cost [27]. Therefore, several studies proposed to use shorter barcode fragments (mini-barcodes) for molecular identification. These sequences often represent the only molecular information available for an insect species and are considered as temporary proxies for the complete DNA barcode. For these reasons, it would be important to quantify which is the proportion of correct identification guaranteed by minibarcodes of different lengths and verify if different regions of the barcode fragment are equally informative in the molecular identification of insects.

The Barcoding of Life Initiative is the main consortium (CBOL) coordinating the collection of DNA barcodes and building a worldwide reference database for the molecular identification of species (BOLD, http:// www.barcodinglife.org). Currently (June 2009) the BOLD system includes $>400,000$ insect DNA barcodes. The validation and/or identification of many of these are still in progress. The "Reference Barcode Database" (RBD) of BOLD aims to reduce the possible biases due to inadequately sampled or misidentified species. Therefore, the RBD only includes validated DNA barcodes of minimally $500 \mathrm{bp}$ and only contains species represented by three or more individuals showing $<2 \%$ sequence divergence [3]. The RDB currently comprises nearly 170,000 insect DNA barcodes from approximately 15,000 species. By far, the largest part of these taxa are lepidopterans ( $>75 \%$ of species), while the remaining $25 \%$ of insect species is distributed among 32 different orders. Although the currently barcoded taxa represent $<2 \%$ of the described insect species, (thus provoking by definition a representativity problem), it is still important to have a first, general estimate of the reliability of insect DNA barcoding in relation to other methodological or conceptual issues. In order to avoid possible biases due to the large proportion of Lepidoptera in the reference database, it would be also important to separately assess the performance of DNA barcoding in different insect orders. After all, if the current DNA barcoding approaches show a low reliability in the molecular identification of insects, then the involved efforts in time, manpower and financial investments [27] could better be re-directed towards complementary or alternative identification methods. Conversely, confirming the reliability of DNA barcoding through a representative insect sample of the BOLD database would support the efforts of all research groups actively involved in insect DNA barcoding.

In this study we assess the reliability of insect DNA barcoding by performing simulations under a "best case scenario" by providing for each query one or more potential conspecific matches in the reference dataset. The objectives of this study were to (1) verify differences in the proportion of correct matches provided in different insect orders by a number of identification criteria, (2) assess if different regions of the barcode fragment are equally informative, (3) investigate relationships between barcode length and identification success, (4) evaluate 
how success is affected by levels of taxon coverage of the reference database.

\section{Results}

The distribution of pairwise K2P distances inferred from the 15,948 DNA barcodes involving 1,995 insect species (Table 1) showed that $95 \%$ of all intraspecific distances were in the interval $0.00-7.64 \%$ and that $95 \%$ of mean interspecific, congeneric distances in the interval 2.47 $21.00 \%$. Both distance distributions were largely overlapping (Figure 1) with $27.32 \%$ of pairwise comparisons shared between the $95 \%$ percentiles of intra- and interspecific distributions $(2.47 \%<\mathrm{K} 2 \mathrm{P}<7.64 \%)$.

The four ID criteria (Best Match: BM; Best Close Match: BCM; All Species Barcodes: ASB; and tree-based identification: NJT) yielded different proportions of correct matches $(\mathrm{NJT}=0.656$, S.D. $=0.118 ; \mathrm{BM}=0.948$, S.D. $=0.026 ; \mathrm{BCM}=0.946$, S.D. $=0.031 ; \mathrm{ASB}=0.796$, S.D. $=$ 0.150 ; Figure 2, Additional files 1, 2). ANOVA revealed highly significant differences among ID criteria and insect orders. A posteriori comparisons of means showed that NJT had always a significantly lower identification success than to both BM and BCM. Regardless the different numbers of taxa sampled in each order, the proportions of correct matches of $\mathrm{BM}$ and $\mathrm{BCM}$ were consistent across Coleoptera (114 species), Diptera (345 species), Hemiptera (164 species), Hymenoptera (160 species), Lepidoptera (1.167 species) and Orthoptera (45 species), while NJT showed significant variations among orders, with the highest proportion of correctly identified queries in Hymenoptera and Orthoptera and the lowest in Diptera (Table 2).

For each identification criterion, regression and pairwise F-tests (Figure 3, Table 3 and 4) did not show significant differences in the proportion of correct matches

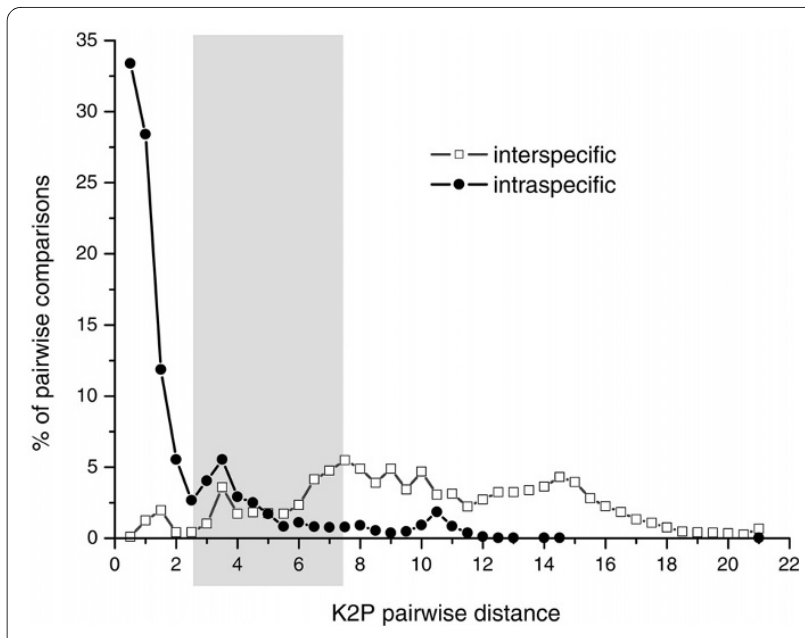

Figure 1 Distance analysis. Distributions of interspecific (white squares) and intraspecific (black circles) pairwise K2P distances resulting from the analysis of 15,948 DNA barcodes belonging to 1,995 insect species (dataset A). In grey: overlap between the $95 \%$ percentiles of intra- and interspecific distributions $(2.47 \%<\mathrm{K} 2 \mathrm{P}<7.64 \%)$

provided by the three mini-barcodes (corresponding to the first, second and last third of the full DNA barcode). Yet, once again there were significant differences between the regression curves obtained through NJT and both $\mathrm{BM}$ and $\mathrm{BCM}$. The proportions of correct identifications obtained from the mini-barcodes of 220 bp were relatively high when using BM $(0.893$, S.D. $=0.013)$ and BCM (0.890, S.D. $=0.013)$, and decreased to $50 \%$ of the value of the full barcode only when very short barcode fragments were considered (40 bp for both $\mathrm{BM}$ and $\mathrm{BCM})$

The identification success of DNA barcoding through $\mathrm{BM}$ and BCM was negatively affected by the numbers of taxa included in the reference dataset, (Figure 4, Table 5), though this effect was remarkably limited as shown by

Table 1: Summary of DNA barcodes considered in this study.

\begin{tabular}{|c|c|c|c|c|c|c|}
\hline & \multicolumn{3}{|c|}{ dataset $A$} & \multicolumn{3}{|c|}{ dataset B } \\
\hline & $\begin{array}{c}\mathbf{n} . \\
\text { barcodes }\end{array}$ & $\begin{array}{c}\text { n. } \\
\text { orders }\end{array}$ & $\begin{array}{c}\text { n. } \\
\text { species }\end{array}$ & $\begin{array}{c}n . \\
\text { barcodes }\end{array}$ & $\begin{array}{c}\text { n. } \\
\text { orders }\end{array}$ & $\begin{array}{c}\text { n. } \\
\text { species }\end{array}$ \\
\hline Coleoptera & 628 & 43 & 114 & 516 & 16 & 58 \\
\hline Diptera & 4,272 & 75 & 345 & 4,010 & 51 & 214 \\
\hline Hemiptera & 901 & 71 & 164 & 745 & 39 & 86 \\
\hline Hymenoptera & 2,067 & 65 & 160 & 1,961 & 43 & 107 \\
\hline Lepidoptera & 7,577 & 495 & 1,167 & 6,567 & 305 & 662 \\
\hline Orthoptera & 503 & 25 & 45 & 455 & 12 & 21 \\
\hline total & 15,948 & 774 & 1,995 & 14,254 & 466 & 1,148 \\
\hline
\end{tabular}

Dataset A (including species represented by at least two DNA barcodes) was used to describe distributions of pairwise distances, to quantify the proportion of correct matches according to Best Match (BM), Best Close Match (BCM), Neighbor-Joining Tree (NJT) and for ANOVA and regression analyses. Dataset $B$ (including species represented by at least three DNA barcodes) was used to estimate the identification success of All Species Barcodes (ASB). 
Table 2: ANOVA and Student-Newman-Keuls (SNK) tests.

\begin{tabular}{lcccc}
\hline Source of variation & $\boldsymbol{d f}$ & MS & $\boldsymbol{F}$ & $\begin{array}{c}\text { denominator MS } \\
\text { for } \boldsymbol{F} \text { ratio }\end{array}$ \\
\hline Criterion $=\mathrm{Cr}$ & 2 & 0.4316 & $34.10^{* * *}$ & $\mathrm{Cr} \times$ Or \\
Order $=$ Or & 5 & 0.0300 & $25.70^{* * *}$ & Residual \\
Cr $\times$ Or & 10 & 0.0168 & & Residual \\
Residual & 36 & 0.0009 & & \\
Total & 53 & & & \\
Cochran's C $=0.270$, not significant & & & &
\end{tabular}

\section{SNK test Cr:}

$\mathrm{BM}=\mathrm{BCM}>\mathrm{NJT}$

\section{SNK test $\mathrm{Cr} \times$ Or:}

$\mathrm{BM}$ : Coleoptera $=$ Diptera $=$ Hemiptera $=$ Hymenoptera $=$ Lepidoptera $=$ Orthoptera

BCM: Coleoptera $=$ Diptera $=$ Hemiptera $=$ Hymenoptera $=$ Lepidoptera $=$ Orthoptera

NJT: Hymenoptera $=$ Orthoptera $>$ Lepidoptera $>$ Coleoptera $>$ Hemiptera $>$ Diptera

Differences in the proportion of correct matches provided by three identification criteria (BM: Best Match, BCM: Best Close Match, NJT: Neighbor-Joining Tree) were tested in six insect orders (Coleoptera, Diptera, Hemiptera, Hymenoptera, Lepidoptera, Orthoptera). Before running the analysis, the assumption of homogeneity of variances was verified using Cochran's C-test. SNK tests were used for $a$ posteriori multiple comparisons of means. df: degrees of freedom, MS: mean squares estimates, ${ }^{* * *}=p<0.001$.

the slope of the regression lines $\left(\mathrm{BM}: \mathrm{b}=-2.13 \times 10^{-5}\right.$, $\left.\mathrm{BCM}: \mathrm{b}=-2.68 \times 10^{-5}\right)$. When passing from 100 to 1,995 species, the proportion of correct matches decreased from 0.998 to 0.948 for $\mathrm{BM}$ and from 0.985 to 0.946 for $\mathrm{BCM}$ (corresponding to $-5.0 \%$ and $-3.9 \%$, respectively).

\section{Discussion}

The relatively high proportion of correctly identified insect taxa by using two simple criteria (BM and $\mathrm{BCM}$ ) provides a general support to the DNA barcoding method per se. These two distance based methods showed comparable results in terms of identification success and performed considerably better than NJT and ASB, which also showed a higher variability in the identification success of the different insect orders (Figure 2). Meier et al. [4] already highlighted the poorer performance of NJT, arguing that this method relies on the topology of one of all the possible Neighbor-Joining trees and does not consider the support of the nodes that separate and define species. Additionally, in case of "ties", NJT can be affected by the input order of taxa $[6,28,29]$. Meier also remarks that queries should be at least one node into monospecific clades, while simply clustering within a clade does not guarantee unambiguous identification [30]. Whether alternative tree reconstruction methods do better than NJT, remains to be decided. For example, Bayesian methods are currently computationally too intensive to be applied to large datasets. Nevertheless, preliminary results show that Bayesian tree reconstruction can provide considerably higher proportions of correct identifications than NJT [6]. On the other hand, the lower identification success of ASB appears related to the more stringent "decision rules" of this criterion (i.e. both the threshold for genetic distances and the need for all the conspecific barcodes of the query as best matches) which could lower the proportion of correct identifications.

Aliabadian et al. [31] stressed that the identification success of distance-based barcoding ultimately depends on the difference between intra- and interspecific divergence and in the ideal world for barcoding there is no overlap between the distributions of these two distance classes. According to Hebert et al. [9] the barcoding gap (i.e. the difference between intra- and interspecific distances) allows to assign specimens to "categories that taxonomists call species" once comprehensive reference libraries of barcodes will be available. Hebert et al. [9] and Aliabadian et al. [31] reported that in birds there is a barcoding gap, such that mean interspecific, congeneric distances are about 20-24 times larger than intraspecific ones. However, growing evidence in birds and other taxa suggests that the overlap between mean intra- and interspecific genetic distances is considerably greater when larger proportions of closely related taxa are included $[13,32]$. Additionally, the extent of the barcoding gap tends to be overestimated when mean intraspecific distances are used, while smallest intraspecific distances 


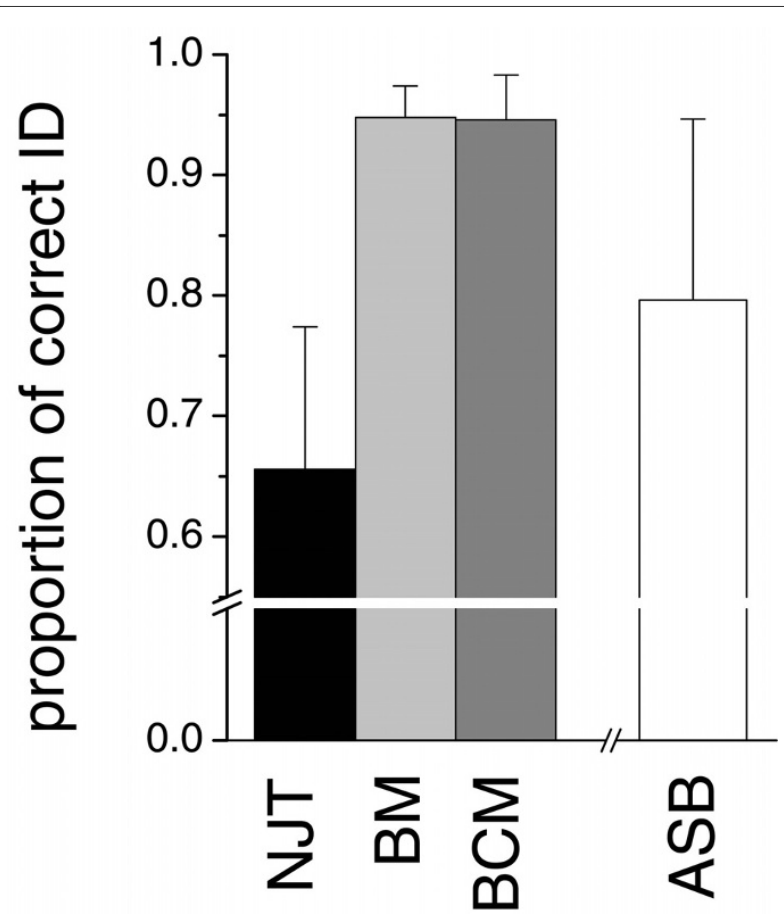

Figure 2 Comparisons between identification criteria for DNA barcoding. Proportion of correctly identified queries through Neighbor-Joining Tree (NJT), Best Match (BM), Best Close Match (BCM) and All Species Barcodes (ASB). For each identification criterion, values were averaged across six insect orders (Coleoptera, Diptera, Hemiptera, Hymenoptera, Lepidoptera, Orthoptera; SD as error bars). Dataset A (including 15,948 DNA barcodes) was used to quantify the proportion of correct identification according to BM, BCM and NJT, while dataset B (including 14,254 DNA barcodes) to quantify the identification success of ASB (see text for explanations).

yield more consistent results [5]. Our data show that the distributions of mean intra- and interspecific congeneric distances of 1,995 insect species are largely overlapping and that the threshold Hebert et al. [9] proposed (mean interspecific, congeneric divergence/mean intraspecific divergence $\geq 10$ ) is not valid to separate insect species. Surprisingly, despite there is no well-defined insect barcoding gap, distance based criteria such as BM and $\mathrm{BCM}$ show a remarkable identification success in our simulations. Hence, although there is a general trend for identification success to decline with increasing overlap between intra- and interspecific distances the extent of the barcoding gap should not be considered as a predictor of the identification success $[5,24,30]$.

Different regions of the full barcode fragment provide comparable information for the molecular identification of insects. Mini-barcodes of $220 \mathrm{bp}$, though less effective than the full barcode, still yield a reasonable insect identification success (about $89 \%$ in our simulations). Though less encouraging than previous simulations based on insect COI fragments of comparable lengths [25,26], our results suggest that mini-barcodes could indeed repre- sent a cost-effective way of building reference databases for the molecular identification of species. However, it would be important to carefully consider the trade offs between mini-barcode length and proportions of correctly identified insect taxa and clearly establish thresholds for the minimal lengths and features of the minibarcodes that will be incorporated into reference databases.

\section{Conclusions}

Although, our simulations show that the probability of false identifications of queries with conspecifics in the reference database is relatively limited (up to $5.2 \%$ when using $\mathrm{BM}$ ), there remains a logical problem with insect DNA barcoding due to the limited proportion of referenced taxa in BOLD and RBD. Indeed, in a real life situation, one does not know in advance whether or not a query is represented in BOLD [4], but one can reasonably assume that there is a good chance that it belongs to the $98 \%$ of insect species that still have to be barcoded. According to $\mathrm{BM}$ (i.e. the identification criterion currently adopted in BOLD) all queries not represented in the reference databases will be, in the best case, assigned to the most closely related taxon available in the database, thus generating a considerable amount of false identifications. Accordingly, whenever the null hypothesis $\mathrm{H}_{0}$ : $\mathrm{Q}_{\mathrm{x}}=$ $\mathrm{Sp}_{\mathrm{A}}$ (where $\mathrm{Q}_{\mathrm{x}}=$ a query about unknown species $\mathrm{X}$ and $\mathrm{Sp}_{\mathrm{A}}=$ species $\mathrm{A}$ ) is accepted, then this may imply either (1) a correct identification of $X$ as species A or (2) a Type II error $\left(\mathrm{H}_{0}=\right.$ accepted when false), where species $\mathrm{X}$ is wrongly identified as A because it is not represented in the reference database and hence is associated with another species. Conversely, if $\mathrm{H}_{0}$ is rejected (i.e. species $\mathrm{X}$ is not $\mathrm{A}$ ), then this may imply either (1) a correct decision that species $\mathrm{X}$ is indeed not $\mathrm{A}$ or (2) a Type I error $\left(\mathrm{H}_{0}=\right.$ rejected when true), where $\mathrm{X}$ is erroneously not recognized as being $\mathrm{A}$. The proportion of Type II errors is hardly predictable, but we should expect higher levels of Type II errors in reference database with poor taxon coverage. Additionally, when using the reference database of BOLD, only part of the queries without conspecifics in the database are misidentified, as the identification procedure is aborted whenever the genetic divergence between query and best match exceeds an arbitrary threshold of 3\% [3]. The simulations performed in this study allowed to quantify the proportion of Type I errors in a large database of insect DNA barcodes. We observed a trend toward a progressive increase of false identifications in databases with larger taxon coverage. When passing from 100 to 1,995 species, the proportion of Type I errors increases of $5.0 \%$ for BM (from $0.2 \%$ to $5.2 \%$ ) and of $3.9 \%$ for BCM (from $1.5 \%$ to $5.4 \%$ ). So, the proportion of correct identifications and the number of species in the 


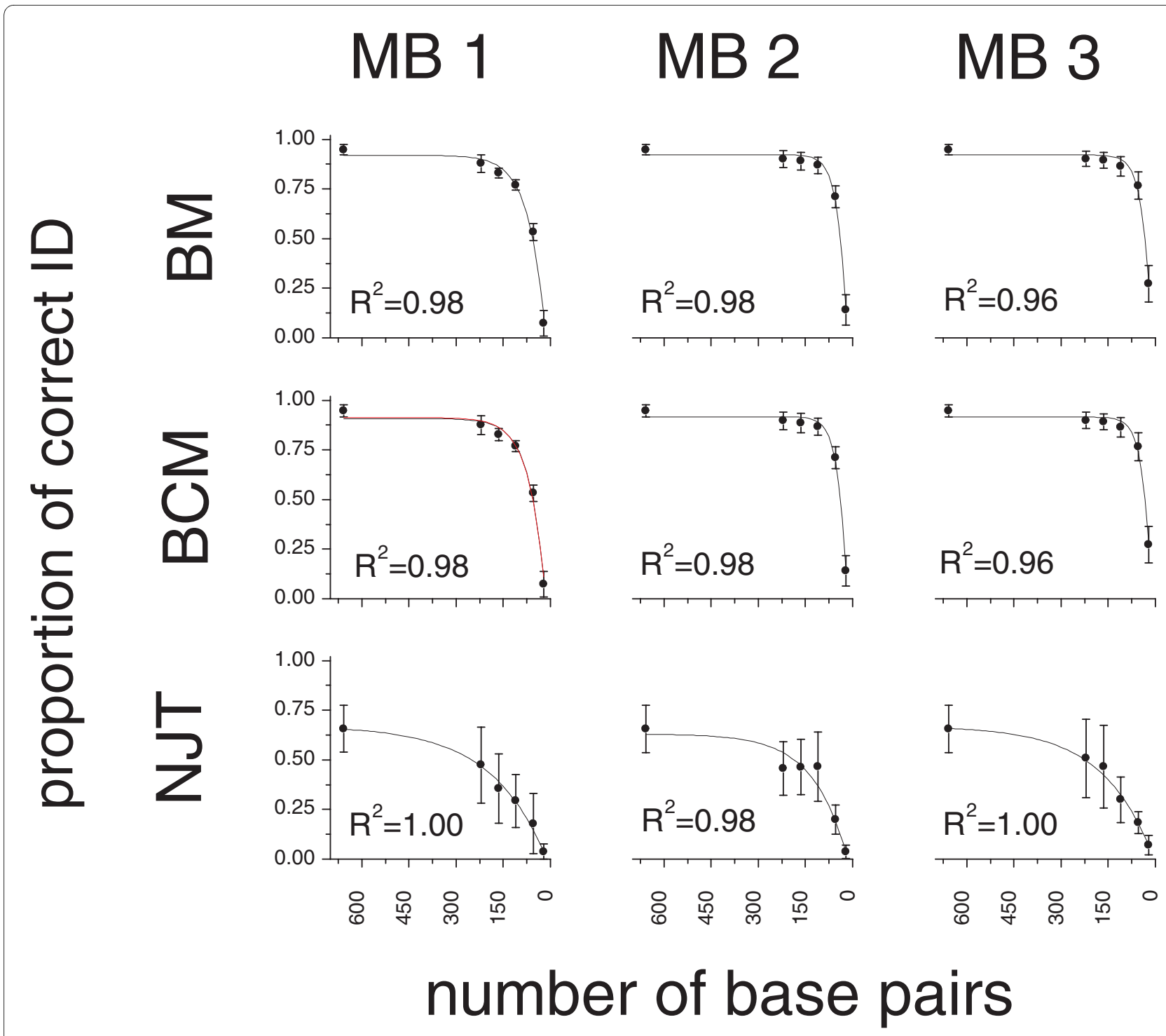

Figure 3 Relationships between barcode length and identification success. Non-linear regression of the identification success obtained by considering the full DNA barcode (550-658 bp) and three non-overlapping "mini-barcodes" (MB1: 220 bp, MB2: 219 bp, MB3: 219 bp). Each mini-barcode was reduced at both 5' and 3' ends in order to obtain fragments of 164 bp, 110 bp, 55 bp and 22 bp (corresponding to approximately to 75\%, 50\%, $25 \%$ and $10 \%$ of the initial mini-barcode length). For each fragment the proportion of correct identifications was calculated according to three identification criteria (BM: Best Match, BCM: Best Close Match, NJT: Neighbor-Joining Tree) and averaged across six insect orders (Coleoptera, Diptera, Hemiptera, Hymenoptera, Lepidoptera, Orthoptera, SD as error bars). Regression curves were fitted following a first order exponential decay model y $=y_{0}+a e^{(-x / t)}$, where $y_{0}=Y$ offset, $a=$ amplitude, $t=$ exponential time constant (see Table 3 ).

reference database are inversely related, even if the slope of the linear regression is close to zero. These results suggest that the combined effects of Type I and II errors can heavily affect the reliability of DNA barcoding in the molecular identification of insects. However, biases related to Type II errors and the limited taxon coverage of the currently available databases could be circumvented by adopting a "negative" approach, i.e. by using DNA barcoding to verify the lack of correspondence between an unknown query and a list of well-known referenced target taxa. This approach would allow ruling out Type II errors and performing a "negative identification" $\left(\mathrm{H}_{0}\right.$ rejected), which is only subject to Type I errors. Given that levels of genetic variability of a group of target species are adequately represented in the reference database, DNA barcoding could hence still be profitably used in quarantine interception of insects of economical importance by indicating that a sample of interest does not include a list of referenced pests. Species of economical significance are generally better characterised from a molecular perspective and are among the best represented in reference databases. Cameron et al. [27] argued 
Table 3: Relationships between barcode length and identification success.

\begin{tabular}{|c|c|c|c|c|c|}
\hline & & $y_{0}$ & A & $\mathbf{t}$ & $\mathbf{R}^{2}$ \\
\hline & MB1 & $\begin{array}{c}0.919 \\
(0.022)\end{array}$ & $\begin{array}{c}1.209 \\
(0.124)\end{array}$ & $\begin{array}{l}53.551 \\
(6.935)\end{array}$ & 0.98 \\
\hline \multirow[t]{3}{*}{ BM } & MB2 & $\begin{array}{c}0.923 \\
(0.019)\end{array}$ & $\begin{array}{c}1.745 \\
(0.377)\end{array}$ & $\begin{array}{l}26.167 \\
(5.663)\end{array}$ & 0.98 \\
\hline & MB3 & $\begin{array}{c}0.922 \\
(0.019)\end{array}$ & $\begin{array}{l}-1.468 \\
(0.486)\end{array}$ & $\begin{array}{l}26.444 \\
(8.277)\end{array}$ & 0.96 \\
\hline & MB1 & $\begin{array}{c}0.913 \\
(0.025)\end{array}$ & $\begin{array}{l}-1.234 \\
(0.133)\end{array}$ & $\begin{array}{l}51.135 \\
(7.286)\end{array}$ & 0.98 \\
\hline \multirow[t]{3}{*}{$\mathrm{BCM}$} & MB2 & $\begin{array}{c}0.917 \\
(0.022)\end{array}$ & $\begin{array}{l}-1.772 \\
(0.398)\end{array}$ & $\begin{array}{l}26.455 \\
(5.716)\end{array}$ & 0.98 \\
\hline & MB3 & $\begin{array}{c}0.915 \\
(0.020)\end{array}$ & $\begin{array}{l}-1.510 \\
(0.525)\end{array}$ & $\begin{array}{l}25.378 \\
(8.234)\end{array}$ & 0.96 \\
\hline & MB1 & $\begin{array}{c}0.671 \\
(0.145)\end{array}$ & $\begin{array}{l}-0.716 \\
(0.138)\end{array}$ & $\begin{array}{c}176.099 \\
(107.271)\end{array}$ & 1.00 \\
\hline \multirow[t]{2}{*}{ NJT } & MB2 & $\begin{array}{c}0.630 \\
(0.116)\end{array}$ & $\begin{array}{l}-0.725 \\
(0.112)\end{array}$ & $\begin{array}{l}108.431 \\
(55.143)\end{array}$ & 0.98 \\
\hline & MB3 & $\begin{array}{c}0.670 \\
(0.136)\end{array}$ & $\begin{array}{l}-0.687 \\
(0.138)\end{array}$ & $\begin{array}{l}160.360 \\
(88.530)\end{array}$ & 1.00 \\
\hline
\end{tabular}

Details of regression analyses of Figure 4. Regression curves were fitted following a first order exponential decay model $y=y_{0}+a e^{(-x / t)}$, where $y_{0}=Y$ offset, $a=$ amplitude, $t=$ exponential time constant. The Goodness-of-fit to the non-linear model is indicated by values of $R^{2}$ close to 1.00 .

that the relatively small number of species that need to be identified for quarantine purposes makes the assembly of a high quality reference database much easier than for all of life and suggested it would be more practical to use barcoding as an entirely DNA based quarantine system. However, it is of crucial importance to understand how the proportion of Type I and Type II errors will change once hundreds of thousands of insect taxa will be included in the reference databases. The lower identification success observed in this study for larger databases should be considered only as a very preliminary indication as the simulations could only include a limited fraction of the global taxonomic diversity of insects (from 100 to 1,995 species). Hence, a major challenge for insect DNA barcoding will be to understand if the error levels associated to the currently adopted distance-based identification criteria will still be acceptable once reference databases will have a more representative coverage of insect taxonomic diversity.

\section{Methods}

We used the DNA barcodes available in June 2009 in BOLD http://www.barcodinglife.org and downloaded all the publicly available sequences belonging to the orders
Coleoptera, Diptera, Hemiptera, Hymenoptera, Lepidoptera and Orthoptera. The dataset was extended with 324 Tephritid (Insecta: Diptera) DNA barcodes collected by the Royal Museum for Central Africa in the framework of the Tephritid Barcoding Initiative, a demonstrator project, initiated by CBOL. DNA barcodes were trimmed in order to include only the barcode region, namely the 658 bp COI fragment amplified by the "universal primers" of Folmer et al. [1]. Sequences shorter than $550 \mathrm{bp}$ and DNA barcodes with incomplete species information (e.g. sequence names including $s p$., $c f$., $n r$. etc.) were discarded. Nucleotide sequences were aligned using the default parameters of the IUB scoring matrix of ClustalW, as implemented in Bioedit 7.0 [33]. Sequences were aligned in blocks of 800 and all the blocks were aligned to a single, haphazardly chosen, profile sequence (accession GQ154187). The blocks were visually inspected to check for possible incongruence (i.e. the occurrence of gaps which were not multiples of $3 \mathrm{bp}$ ). Each block was then pasted into the data matrix until all sequences were aligned. In order to allow comparisons between our results and large part of the current literature on DNA barcoding, we used pairwise Kimura's two parameter (K2P) distances [34] and plotted the frequency 
Table 4: Pairwise comparisons among non-linear regression plots of Figure 2.

\begin{tabular}{|c|c|c|c|c|c|c|c|c|c|c|}
\hline & & & BM & & & $\mathrm{BCM}$ & & & NJT & \\
\hline & & MB1 & MB2 & MB3 & MB1 & MB2 & MB3 & MB1 & MB2 & MB3 \\
\hline & MB1 & & 2.348 & 4.307 & 0.001 & 2.307 & 4.423 & 43.972 & 7.433 & 44.186 \\
\hline \multirow[t]{3}{*}{ BM } & MB2 & n.s. & & 0.831 & 2.476 & 0.003 & 0.864 & 80.475 & 11.491 & 91.195 \\
\hline & MB3 & n.s. & n.s. & & 4.477 & 0.838 & 0.001 & 72.996 & 12.405 & 78.593 \\
\hline & MB1 & n.s. & n.s. & n.s. & & 2.430 & 4.600 & 44.745 & 7.375 & 45.241 \\
\hline \multirow[t]{3}{*}{$\mathrm{BCM}$} & MB2 & n.s. & n.s. & n.s. & n.s. & & 0.867 & 79.447 & 11.344 & 89.818 \\
\hline & MB3 & n.s. & n.s. & n.s. & n.s. & n.s. & & 75.877 & 12.440 & 82.774 \\
\hline & MB1 & $* *$ & $* * *$ & $* * *$ & $* *$ & $* * *$ & $* * *$ & & 0.628 & 1.089 \\
\hline \multirow[t]{2}{*}{ NJT } & MB2 & * & * & * & * & * & * & n.s. & & 0.190 \\
\hline & MB3 & $* *$ & $* * *$ & $* * *$ & $* *$ & $* * *$ & $* * *$ & n.s. & n.s. & \\
\hline
\end{tabular}

distributions of intraspecific and mean interspecific, congeneric distances.

We estimated the proportion of correct matches provided by the four identification criteria described by Meier et al. [4]. These included three distance-based criteria (Best Match: BM; Best Close Match: BCM; All Species Barcodes: ASB) and the tree-based identification (tree-identification: NJT). According to BM, each query was assigned the species name of its best-matching barcode regardless of how similar the query and barcode sequences were. In our simulation, identification was considered successful when both sequences were from the same species. BCM relies on a threshold value of sequence similarity. The threshold was estimated from dataset A (see below) by establishing a frequency distribution of pairwise intraspecific distances and determining the distance below which $95 \%$ of all intraspecific distances are found. BCM first identified the best barcode match of a query and then assigned the species name of that barcode only if the distance between query and barcode was below the threshold. The simulation of ASB proceeded as for BM but assigned a species name only when all the conspecifics of the query topped the list of best matches. NJT considered an identification to be correct if the query and all its conspecific sequences formed a monospecific clade. We evaluated the identification success (i.e. the proportion of correct matches) of the four criteria by using two separate datasets (Table 1): (1) species of which a query would find at least one conspecific match in the reference database (dataset A, 15,948 DNA barcodes, 1,995 species, Additional file 3) and (2) species of which a query would find at least two conspecific matches in the reference database (dataset B, 14,254 DNA barcodes, 1,148 species, Additional file 4). Dataset A was used to evaluate the proportion of correct matches according to BM, BCM and NJT, while dataset B to evaluate the identification success of ASB. SpeciesIdentifier1.5 [4] was used to calculate pairwise $\mathrm{K} 2 \mathrm{P}$ distances and to quantify the proportion of correct matches according to $\mathrm{BM}, \mathrm{BCM}$ and ASB. The identification success of NJT was quantified using PAUP* [35] to reconstruct a Neighbor-Joining tree (K2P distance, ties randomly broken)

Table 5: Relationships between taxon coverage of the reference database and identification success.

\begin{tabular}{|c|c|c|c|}
\hline BM & & BCM & \\
\hline $\mathrm{R}^{2}=1.00$ & & $R^{2}=0.99$ & \\
\hline $\mathrm{F}=651.91^{* * *}$ & & $\mathrm{~F}=236.178^{* * *}$ & \\
\hline$a=0.986(0.001)$ & $\mathrm{t}=961.4747^{* * *}$ & $a=0.999(0.002)$ & $\mathrm{t}=465.262^{* * *}$ \\
\hline$b=-2.13 \times 10^{-5}\left(1.74 \times 10^{-6}\right)$ & $\mathrm{t}=-25.53262^{* * *}$ & $b=-2.68 \times 10^{-5}\left(1.06 \times 10^{-6}\right)$ & $t=-15.368^{* * *}$ \\
\hline
\end{tabular}

Details of regression analyses of Figure 3 . Regression lines were fitted according to the linear model $y=a+b x($ where $a=y$-intercept, $b=$ slope). Goodness-of-fit is indicated by values of $R^{2}$ close to 1.00 and by the statistical significance of (1) F-test of the overall fit, (2) t-tests of individual parameters. ${ }^{* *}: p<0.001$. 


\section{BM

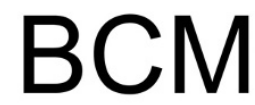

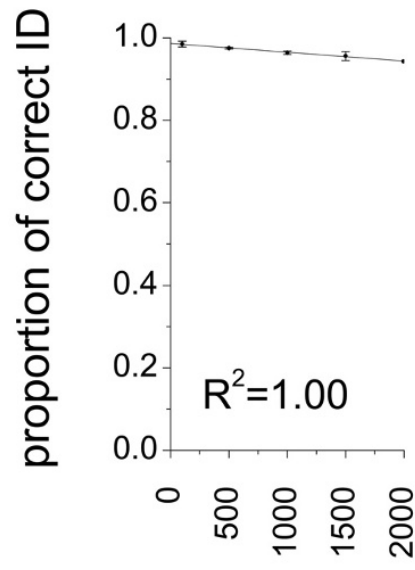

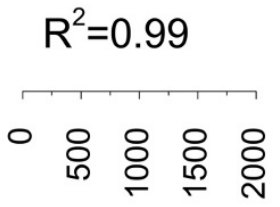

\section{n. of species}

Figure 4 Relationships between taxon coverage of the reference database and identification success. Databases including 100, 500, 1,000 and 1,500 insect species were obtained by randomly sampling dataset A (three replicates for each subset of species). The identification success of Best Match (BM) and Best Close Match (BCM) was averaged across replicates (SD as error bars) and linear regression fitted after including the proportion of correct matches resulting from the analysis of the whole dataset (1,995 species).

and calculating the NJT identification success by using a Perl script developed for this purpose (TreeCode.v1, Additional file 5).

Differences in the identification success of (1) BM, BCM and NJT and (2) Coleoptera, Diptera, Hemiptera, Hymenoptera, Lepidoptera, Orthoptera were tested by a 2-way model of analysis of variance (ANOVA). ANOVA was based on three independent replicates, sensu Underwood [36], which were obtained by (1) randomly assigning the DNA barcodes of each order to three groups (each corresponding to an identification criterion) and (2) dividing each group in three sub-groups. Each replicate was represented by the proportion of correct matches calculated in each sub-group, according to the identification criterion of each group. Identification criterion $(\mathrm{Cr}, 3$ levels, fixed) and insect order (Or, 6 levels, random) were considered as orthogonal variables. The assumption of homogeneity of variances was verified through Cochran's C-test. Student-Newman-Keuls (SNK) tests were used for a posteriori multiple comparisons of means [36]. The identification success of ASB (calculated from dataset B) was qualitatively compared with the proportion of correct matches obtained for $\mathrm{BM}, \mathrm{BCM}$ and NJT (calculated from dataset A).
Relationships between barcode length and identification success were analysed through non-linear regression. The DNA barcodes of dataset A were divided in three non-overlapping "mini-barcodes" of 220, 219 and $219 \mathrm{bp}$ corresponding to the first, second and last third of the barcode region (hereafter MB1, MB2, MB3). The number of bp of each mini-barcode was further reduced at both $5^{\prime}$ and 3' ends in order to obtain fragments of approximately $75 \%, 50 \%, 25 \%$ and $10 \%$ of the initial mini-barcode length (164 bp, 110 bp, 55 bp and 22 bp, respectively). For each fragment the proportion of correct identifications was calculated according to three identification criteria (BM, $\mathrm{BCM}, \mathrm{NJT}$ ) and averaged across the six insect orders (Coleoptera, Diptera, Hemiptera, Hymenoptera, Lepidoptera, Orthoptera). Non-linear regression fitting was implemented for each combination of identification criterion and barcode fragment following the first order exponential decay model $y=y_{0}+a e^{(-x / t)}$, where $y_{0}=Y$ offset, a $=$ amplitude, $\mathrm{t}=$ exponential time constant [37]. Differences among regression curves were verified through pairwise F-tests. Probability values of repeated comparisons were corrected for Type I errors using the False Discovery Rate procedure [38]. Relationships between levels of taxon coverage in the reference database and identification success were investigated through linear regression. The DNA barcodes of dataset A were randomly sampled in order to obtain reference databases including subsets of 100, 500, 1000 and 1,500 insect species. Three independent replicates were obtained for each subset. The identification success of BM and BCM was averaged across the three replicates of each subset and regressions were fitted after including the proportion of correct matches resulting from the analysis of the whole dataset (1,995 species). Regression analyses were implemented in OriginPro v7 http://www.originlab.com after comparing the Goodness-of-fit of alternative models.

\section{Additional material}

\section{Additional file 1 Summary of simulations on Dataset $A$.}

Additional file 2 Summary of simulations on Dataset $B$.

Additional file 3 Dataset A. Accession numbers and alignment of 15,948 DNA barcodes (>550 bp) belonging to 1,995 insect species, each represented by at least two sequences.

Additional file 4 Dataset B. Accession numbers and alignment of 14,254 DNA barcodes (>550 bp) belonging to 1,148 insect species, each represented by at least three sequences.

Additional file $\mathbf{5}$ TreeCode.1.0. Perl script developed to quantify the proportion of correctly identified queries according to the NJT criterion.

\section{Authors' contributions}

MV conceived the study, performed the statistical analyses and drafted the manuscript. BN developed the bioinformatic tools and participated to the analysis of data and manuscript preparation. TB and MDM contributed to the study design and critically reviewed the manuscript. All authors read and approved the final version of the manuscript. 


\section{Acknowledgements}

We are grateful to Zoltan Tamas Nagy, Céline Poux and Erik Verheyen for stimulating discussions on an earlier draft of this manuscript. Analyses of the public DNA barcodes of BOLD were possible thanks to the kind collaboration and expertise of Megan Milton. This study was conducted within the framework of the Tephritid Barcoding Initiative (a demonstrator project, initiated by CBOL) and benefited from practical support by Karin Breugelmans and the "Joint Experimental Molecular Unit (JEMU)" of RBINS and RMCA. We wish to thank David Balata and Fabio Bulleri for comments and suggestions on statistical analyses. MV was funded by the Belgian Science Policy Action 1 (project MO/ 37/017). BN was supported by PhD grant SFRH/BD/17704/2004 from the Fundação para a Ciência e Tecnologia.

\section{Author Details}

1Royal Museum for Central Africa, Leuvensesteenweg 13, 3080, Tervuren, Belgium, 2Royal Belgian Institute of Natural Sciences, Vautierstraat 29, 1000, Brussels, Belgium and ${ }^{3}$ Department of Biology, University of Antwerp, Groenenborgerlaan 171, B-2020 Antwerp, Belgium

Received: 25 September 2009 Accepted: 27 April 2010

Published: 27 April 2010

\section{References}

1. Folmer O, Black M, Hoeh W, Lutz R, Vrijenhoek R: DNA primers for amplification of mitochondrial cytochrome $C$ oxidase subunit I from diverse metazoan invertebrates. Mol Mar Biol Biotechnol 1994, 3:294-299.

2. Hebert PDN, Cywinska A, Ball SL, deWaard JR: Biological identifications through DNA barcodes. Proc $R$ Soc B 2003, 270:313-321.

3. Ratnasingham S, Hebert P: BOLD: The Barcode of Life Data System. Mol Ecol Notes 2007, 7:355-364 [http://www.barcodinglife.org].

4. Meier R, Shiyang K, Vaidya G, Ng PKL: DNA barcoding and taxonomy in Diptera: a tale of high intraspecific variability and low identification success. Syst Biol 2006, 55:715-728.

5. Meier R, Zhang G, Ali F: The use of mean instead of smallest interspecific distances exaggerates the size of the barcoding gap and leads to misidentification. Syst Biol 2008, 57:809-813.

6. Munch K, Boomsma W, Huelsenbeck JP, Willerslev E, Nielsen R: Statistical assignment of DNA sequences using Bayesian phylogenetics. Syst Biol 2008, 57:750-757.

7. Little DP, Stevenson DW: A comparison of algorithms for the identification of specimens using DNA barcodes: examples from gymnosperms. Cladistics 2007, 23:1-21.

8. Hebert PDN, Penton EH, Burns JM, Janzen DH, Hallwachs W: Ten species in one: DNA barcoding reveals cryptic species in the neotropical skipper butterfly Astraptes fulgerator. Proc Natl Acad Sci USA 2004, 101:14812-14817.

9. Hebert PDN, Stoeckle MY, Zemlak TS, Francis CM: Identification of birds through DNA barcodes. PLoS Biol 2004, 2:e312.

10. Hebert PDN, Gregory TR: The promise of DNA barcoding for taxonomy. Syst Biol 2005, 54:852-859.

11. Smith MA, Rodriguez JJ, Whitfield JB, Deans AR, Janzen DH, Hallwachs W, Hebert PDN: Extreme diversity of tropical parasitoid wasps exposed by iterative integration of natural history, DNA barcoding, morphology, and collections. Proc Natl Acad Sci USA 2008, 105:12359-12364.

12. Hajibabaei M, Janzen DH, Burns JM, Hallwachs W, Hebert PDN: DNA barcodes distinguish species of tropical Lepidoptera. Proc Natl Acad Sci USA 2006, 103:968-971.

13. Funk DJ, Omland KE: Species-level paraphyly and polyphyly: frequency, causes, and consequences, with insights from animal mitochondrial DNA. Annual Review of Ecology, Evolution, and Systematics 2003, 34:397-423.

14. Whitworth TL, Dawson RD, Magalon H, Baudry E: DNA barcoding cannot reliably identify species of the blowfly genus Protocalliphora (Diptera: Calliphoridae). Proc R Soc B 2007, 274:1731-1739.

15. Foottit RG, Adler PH, Eds: Insect biodiversity, science and society. Chichester, UK: Wiley-Blackwell; 2009.

16. Burns JM, Janzen DH, Hajibabaei M, Hallwachs W, Hebert PDN: DNA barcodes and cryptic species of skipper butterflies in the genus Perichares in Area de Conservacion Guanacaste, Costa Rica. Proc Natl Acad Sci USA 2008, 105:6350-6355.
17. Fisher BL, Smith MA: A revision of Malagasy species of Anochetus Mayr and Odontomachus Latreille (Hymenoptera: Formicidae). PLOS ONE 2008, 3:e1787.

18. Greenstone MH, Rowley DL, Heimbach U, Lundgren JG, Pfannenstiel RS Rehner SA: Barcoding generalist predators by polymerase chain reaction: carabids and spiders. Mol Ecol 2005, 14:3247-3266.

19. Smith MA, Wood DM, Janzen DH, Hallwachs W, Hebert PDN: DNA barcodes affirm that 16 species of apparently generalist tropical parasitoid flies (Diptera, Tachinidae) are not all generalists. Proc Natl Acad Sci USA 2007, 104:4967-4972.

20. Smith MA, Woodley NE, Janzen DH, Hallwachs W, Hebert PDN: DNA barcodes reveal cryptic host-specificity within the presumed polyphagous members of a genus of parasitoid flies (Diptera: Tachinidae). Proc Natl Acad Sci USA 2006, 103:3657-3662.

21. Elias M, Hill RI, Willmott KR, Dasmahapatra KK, Brower AVZ, Malllet J, Jiggins CD: Limited performance of DNA barcoding in a diverse community of tropical butterflies. Proc R SOC B 2007, 274:2881-2889.

22. Trewick SA: DNA Barcoding is not enough: mismatch of taxonomy and genealogy in New Zealand grasshoppers (Orthoptera: Acrididae). Cladistics 2007, 23:1-5.

23. Ward RD, Hanner R, Hebert PDN: The campaign to DNA barcode all fishes, FISH-BOL. J Fish Biol 2009, 74:329-356.

24. Ross HA, Murugan S, Sibon Li WL: Testing the reliability of genetic methods of species identification via simulation. Syst Biol 2008, 57:216-230

25. Hajibabaei M, Smith MA, Janzen DH, Rodriguez JJ, Whitfield JB, Hebert PDN: A minimalist barcode can identify a specimen whose DNA is degraded. Mol Ecol Notes 2006, 6:959-964.

26. Meusnier I, Singer G, Landry J-F, Hickey D, Hebert P, Hajibabaei M: A universal DNA mini-barcode for biodiversity analysis. BMC Genomics 2008, 9:214

27. Cameron $S$, Rubinoff $D$, Will $K$ : Who will actually use DNA barcoding and what will it cost? Syst Biol 2006, 55:844-847.

28. Farris JS, Albert VA, Källersjö M, Lipscomb D, Klug AG: Parsimony jackknifing outperforms Neighbor-joining. Cladistics 1996, 12:99-124.

29. Backeljau T, De Bruyn L, De Wolf H, Jordaens K: Multiple UPGMA and Neighbor-joining trees and the performance of some computer packages. Mol Biol Evol 1996, 13:309-313.

30. Meier R: DNA sequences in taxonomy, opportunities and challenges. In The new taxonomy Edited by: Wheeler QD. Boca Raton: CRC Press; 2008:95-128.

31. Aliabadian M, Kaboli M, Nijman V, Vences M: Molecular identification of birds: performance of distance-based DNA barcoding in three genes to delimit parapatric species. PLOS ONE 2009, 4:e4119.

32. Moritz C, Cicero C: DNA barcoding: promise and pitfalls. PLOS Biol 2004, 2:e354.

33. Hall TA: BioEdit: a user-friendly biological sequence alignment editor and analysis program for Windows 95/98/NT. Nucleic Acids Symp Ser 1999, 41:95-98.

34. Kimura M: A simple method for estimating evolutionary rate of base substitution through comparative studies of nucleotide sequences. Mol Evol 1980, 16:111-120.

35. Swofford DL: Phylogenetic Analysis Using Parsimony (and other methods). Version 4. Sinauer Associates, Sunderland, Massachusetts; 2002

36. Underwood AJ: Experiments in ecology Cambridge: Cambridge University Press; 1997

37. Sokal RR, Rohlf FJ: Biometry: the principles and practice of statistics in biological research New York: Freeman Press; 1995

38. Benjamini Y, Hochberg Y: Controlling the false discovery rate: a practical and powerful approach to multiple testing. Journal of the Royal Statistical Society series B-statistical methodology 1995, 57:289-300.

doi: $10.1186 / 1471-2105-11-206$

Cite this article as: Virgilio et al., Comparative performances of DNA barcoding across insect orders BMC Bioinformatics 2010, 11:206 